\title{
1 Phage origin of mitochondrion-localized family A DNA 2 polymerases in kinetoplastids and diplonemids
}

4 Ryo Harada ${ }^{1} \&$ Yuji Inagaki ${ }^{1,2, *}$

5

6 'Graduate School of Life and Environmental Sciences, University of Tsukuba, Japan.

$7 \quad{ }^{2}$ Center for Computational Sciences, University of Tsukuba, Japan.

9 *Author for Correspondence: Yuji Inagaki, Center for Computational Sciences,

10 University of Tsukuba, Tsukuba, Japan. Phone: +8129853 6483, Fax: +81 29853

11 6404, Email: yuji@ccs.tsukuba.ac.jp 


\section{Abstract}

13 Mitochondria retain their own genomes as other bacterial endosymbiont-derived

14 organelles. Nevertheless, no protein for DNA replication and repair is encoded in any

15 mitochondrial genomes (mtDNAs) assessed to date, suggesting the nucleus

16 primarily governs the maintenance of mtDNA. As the proteins of diverse evolutionary

17 origins occupy a large proportion of the current mitochondrial proteomes, we

18 anticipate finding the same evolutionary trend in the nucleus-encoded machinery for

19 mtDNA maintenance. Indeed, none of the DNA polymerases (DNAPs) in the

20 mitochondrial endosymbiont, a putative a-proteobacterium, seemingly had been

21 inherited by their descendants (mitochondria), as none of the known types of

22 mitochondrion-localized DNAP showed a specific affinity to the a-proteobacterial

23 DNAPs. Nevertheless, we currently have no concrete idea of how and when the

24 known types of mitochondrion-localized DNAPs emerged. We here explored the

25 origins of mitochondrion-localized DNAPs after the improvement of the samplings of

26 DNAPs from bacteria and phages/viruses. Past studies revealed that a set of

27 mitochondrion-localized DNAPs in kinetoplastids and diplonemids, namely PollB,

28 PollC, PollD, Poll-Perk1/2, and Poll-dipl (henceforth designated collectively as

29 "PollBCD+") have emerged from a single DNAP. In this study, we recovered an

30 intimate connection between PollBCD+ and the DNAPs found in a particular group of

31 phages. Thus, the common ancestor of kinetoplastids and diplonemids most likely

32 converted a laterally acquired phage DNAP into a mitochondrion-localized DNAP

33 that was ancestral to PollBCD+. The phage origin of PollBCD+ hints at a potentially

34 large contribution of proteins acquired via non-vertical processes to the machinery

35 for mtDNA maintenance in kinetoplastids and diplonemids.

37 Keywords: DNA replication, DNA repair, autographivirus, Euglenozoa, lateral gene

38 transfer, mitochondria

\section{Introduction}

40 Mitochondria in the extant eukaryotes are the descendants of an

41 endosymbiotic a-proteobacterium in the last eukaryotic common ancestor (Roger et

42 al. 2017). The mitochondrial (mt) proteins, which are localized in mitochondria, are 
43 almost entirely nucleus-encoded and evolutionarily multifarious (Gabaldón and 44 Huynen 2007; Wang and Wu 2014; Gray 2015). Only 10-20\% of mt proteins were

45 predicted to be of the a-proteobacterial origin, suggesting that the original proteome 46 of the mitochondrial endosymbiont has been remodeled largely (Gray 2015). There

47 are three possible evolutionary paths that coopt non- $\alpha$-proteobacterial proteins into 48 the molecular machinery in mitochondria. Non-a-proteobacterial mt proteins could 49 emerge (i) de novo, (ii) by recycling of the pre-existing eukaryotic proteins, or (iii) via 50 lateral gene transfer. Mitochondria, in principle, retain their own genomes that have 51 been descended from the mitochondrial endosymbiont, albeit the entire set of 52 proteins required for mtDNA maintenance (replication and repair) is nucleus53 encoded. Thus, as a part of the mitochondrial proteome, the machinery for mtDNA 54 maintenance may be dominated by non-a-proteobacterial proteins. Indeed, none of 55 the known DNA polymerases (DNAPs) localized in mitochondria is most unlikely the 56 direct descendant of the DNAPs in the a-proteobacterial endosymbiont that gave rise to the ancestral mitochondrion (see below).

Phylogenetically diverse eukaryotes possess family A (famA) DNAPs that are 59 evolutionarily related to DNA polymerase I (Poll) in bacteria (Jung et al. 1987;

60 Moriyama et al. 2011). Some of famA DNAPs in eukaryotes are known to be 61 localized in mitochondria (Krasich and Copeland 2017). So far, four distinct types of 62 mitochondrion-localized famA DNAP have been identified. First, "plant and protist 63 organellar DNA polymerase (POP)" appeared to be broadly distributed among 64 eukaryotes (Moriyama et al. 2011; Hirakawa and Watanabe 2019). Second, animals 65 and fungi are known to use DNA polymerase gamma (Poly) for mtDNA maintenance 66 (Graziewicz et al. 2006). The third type of mitochondrion-localized famA DNAP is 67 "PollA" shared among members of the classes Kinetoplastea, Diplonemea, and 68 Euglenida, which comprise the phylum Euglenozoa (Klingbeil et al. 2002; Harada et 69 al. 2020). Members of Kinetoplastea and Diplonemea possess the fourth type of 70 mitochondrion-localized famA DNAP. "PollB," "PolIC," and "PolID" were reported originally from a model kinetoplastid Trypanosoma brucei, and later identified in broad members of Kinetoplastea (Klingbeil et al. 2002; Harada et al. 2020). The three DNAPs were shown to be closely related to one another in phylogenetic analyses. A recent study further identified multiple DNAPs, which are closely related to but distinct from PollB, C, or D, in an early-branching kinetoplastid Perkinsela sp. and diverse diplonemids (Poll-Perk1/2 and Poll-dipl; Harada et al. 2020). PollB, C, D, 
77 and their related DNAPs were derived from a single molecule, and thus can be

78 regarded collectively as the fourth type of mitochondrion-localized famA DNAP

79 (henceforth termed as "PollBCD+" in this study). Pioneering studies considered none

80 of the known mitochondrion-localized famA DNAPs as the direct descendant of Poll

81 in the mitochondrial endosymbiont, but failed to clarify how and when POP, Poly,

82 PollA, and PollBCD+ were established in eukaryotic evolution (Moriyama et al. 2011;

83 Hirakawa and Watanabe 2019; Harada et al. 2020).

84 In this study, we explored the origins of mitochondrion-localized famA DNAPs

85 by analyzing an improved dataset wherein sequence sampling from bacteria and

86 phages was improved drastically. We recovered the intimate affinity between

87 PollBCD+ and the famA DNAPs of a particular group of phages in phylogenetic

88 analyses. Furthermore, these DNAPs appeared to share a unique insertion of

89 consecutive 8 amino acid (aa) residues. Altogether, we conclude that the extent

90 DNAPs belonging to PollBCD+ were derived from a single phage famA DNAP

91 acquired by the common ancestor of Kinetoplastea and Diplonemea. We also

92 propose that PollA in Euglenozoa emerged from a type of cytosolic famA DNAP

93 (Pol $\theta$ ). The origins of PollA and PollBCD+ maybe a tip of the remodeling of the

94 machinery of mtDNA maintenance undergone in Kinetoplastea and Diplonemea.

95 Results

96 Prior to this study, the origin of none of the four types of mitochondrion-

97 localized famA DNAPs (i.e. Poly, POP, PollA, and PollBCD+) has been elucidated in

98 detail. This study successfully clarified the origin of PollBCD+ by analyzing

99 phylogenetic alignments that are much richer in bacterial and phage famA DNAPs

100 than those analyzed in the past studies. The sampling of the bacterial homologs was

101 insufficient to reflect the diversity of bacteria in the previously published phylogenies

102 of famA DNAPs (Moriyama et al. 2011; Hirakawa and Watanabe 2019; Harada et al.

103 2020). Furthermore, only a few famA DNAPs of phages have been included in the

104 phylogenetic analyses. In this study we prepared the "global famA DNAP" alignment

105 by incorporating diverse bacterial and phage sequences (446 in total) deposited in

106 public databases and 27 sequences that represent the four mitochondrion-localized

107 types of DNAPs (Poly, POP, PollA, and PollBCD+), a single cytosolic DNAP (Pole), 
108 and a single plastid-localized DNAP found exclusively in apicomplexans and 109 chrompodellids (PREX).

110 The global famA DNAP phylogeny reconstructed four clades, all comprising

111 the eukaryotic homologs exclusively: (i) POP, (ii) PollA plus Pole, (iii) PREX, and (iv)

112 Poly (Shaded in blue in Fig. 1A; see the supplementary materials for the tree with

113 sequence names). The maximum likelihood bootstrap values (MLBPs) for the four

114 clades varied between from 69 to 100\%. The POP, PollA plus Pole, or Poly

115 sequences showed no clear affinity to any bacterial or phage famA DNAPs, leaving

116 their origins uncertain. The PREX sequences grouped with bifunctional 3'-5'

117 exonuclease/DNA polymerases in phylogenetically limited bacteria as previously

118 reported (Janouškovec et al. 2015; Hirakawa and Watanabe 2019; Harada et al.

119 2020). Curiously, the PollBCD+ sequences were paraphyletic but nested within a

120 robustly supported clade mainly comprising famA DNAP homologs of phages

121 belonging to families Autographiviridae and Podoviridae (Fig. 1B; this figure

122 corresponds to the portion shaded in gray in Fig. 1A). The famA DNAP homologs of

123 autographiviruses and three bacteria formed a subclade with an MLBP of $72 \%$. The

124 coding regions of two out of the three bacterial famA DNAP homologs in this

125 subclade (marked by stars in Fig. 1B) are flanked by phage-like open reading frames

126 (ORFs) in the corresponding genome assemblies deposited under the GenBank

127 accession Nos LEDQ01000001.1 and NZ_LLYA01000167.1. Phage-like ORFs

128 including that of famA DNAP encompass $>40 \mathrm{Kbp}$ consecutively in the two bacterial

129 genomes. Thus, the two "bacterial famA DNAPs" are most likely of lysogenic

130 autographiviruses in bacterial genomes. On the other hand, no phage-like ORF was

131 found around that of famA DNAP in the genome of Bordetella genomosp. 9 strain

132 AU14267 (NZ_CP021109.1), suggesting that this bacterium acquired a famA DNAP

133 gene from an autographivirus horizontally. The four PollBCD+ sequences were

134 positioned at the base of the Autographiviridae clade described above and the

135 grouping of PollBCD+ sequences and autographivirus famA DNAPs as a whole

136 received an MLBP of 99\% (Fig. 1B). The global famA DNAP phylogeny strongly

137 suggests an intimate evolutionary affinity between PollBCD+ and autographivirus

138 famA DNAPs.

139 


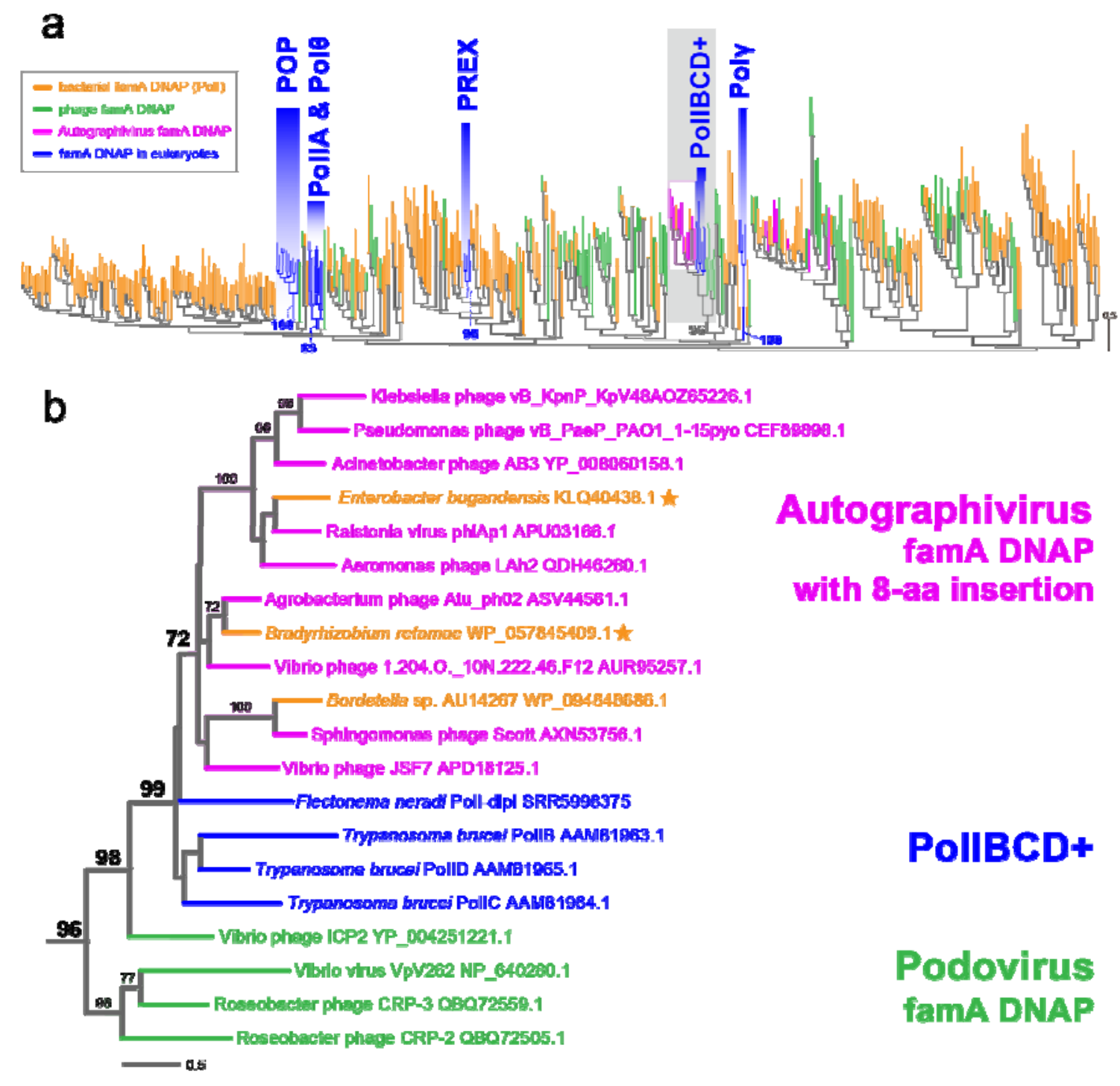

Fig. 1. Maximum likelihood (ML) phylogenetic tree inferred from an alignment of the fam $A$ DNAP sequences of bacteria, phages/viruses, and eukaryotes. (A) Overview of the entire ML tree. All of the sequence names are omitted. The bacterial and eukaryotic sequences are shown in orange and blue, respectively. The sequences of autographiviruses are shown in magenta. A subset of autographiviruses possess famA DNAPs in the pink-shaded clade bears the characteristic insertion of 8 amino acid residues $\left(A G V^{\text {tins }}\right.$ famA DNAPs; see the main text for the details). Other phage/viral sequences are shown in green. Only ML bootstrap values of interest are shown. The subtree containing PollBCD+ and $A G V^{\text {tins }}$ famA DNAP sequences (shaded in gray) is enlarged and presented as (B). ML bootstrap values greater than $70 \%$ are shown. $\mathrm{AGV}^{\text {ins }}$ fam $\mathrm{A}$ DNAP sequences marked by stars are of the putative lysogenic phages in bacterial genomes. structures and possess double-stranded linear DNA genomes of approximately 41

$155 \mathrm{Kbp}$ in length. This viral family comprises 9 subfamilies and 132 genera (Lavigne et al. 2008; Adriaenssens et al. 2020). We searched for autographivirus famA DNAPs 
157 in the GenBank nr database and detected 175 homologs of 99 members belonging

158 to 57 genera and 76 unclassified members. Each of the 175 members of

159 Autographiviridae seemingly possesses a single famA DNAP. Intriguingly, the

160 autographivirus famA DNAPs were split into two types based on the

161 presence/absence of "8-aa insertion" in the polymerase domain (Fig. S1 and Table

162 S1). In this study, we designate autographivirus famA DNAPs with 8-aa insertion as

163 "AGV ${ }^{+ \text {ins }}$ famA DNAPs". Each $\mathrm{AGV}^{+ \text {ins }}$ famA DNAPs was predicted to possess only

164 polymerase domain by InterProScan5 with the Pfam database (Jones et al. 2014; El-

165 Gebali et al. 2019) (Table. S2). AGV ${ }^{\text {ins }}$ famA DNAPs were found in 40 members

166 belonging to 23 genera, and 51 unclassified members (Fig. S1). Although only a

167 subset of the 175 autographivirus famA DNAPs was included, the global famA DNAP

168 phylogeny (Fig. 1A) demonstrated the distant relationship between $A_{G V}{ }^{+i n s}$ famA

169 DNAPs and other autographivirus famA DNAPs lacking 8-aa insertions.

170 To reexamine the phylogenetic affinity between PollBCD+ and $A G V^{+i n s}$ famA

171 DNAPs, we selected non-redundant sequences from the $91 \mathrm{AGV}^{+ \text {ins }}$ famA DNAPs

172 and aligned with 24 PollBCD+ sequences and four famA DNAPs of phages

173 belonging to a family Podoviridae as the outgroup. The second famA DNAP

174 alignment was subjected to both $\mathrm{ML}$ and Bayesian methods. In the second

175 phylogenetic analyses, AGV $^{+ \text {ins }}$ famA DNAPs and PollBCD+ sequences formed a

176 clade supported by an MLBP of $100 \%$ and a Bayesian posterior probability (BPP) of

1771.0 (Fig. 2). PollBCD+ sequences appeared to possess 8 amino acids that are most

178 likely homologous to 8-aa insertion in $A_{G V}^{\text {+ins }}$ famA DNAPs (Fig. 2), strengthening

179 the phylogenetic affinity between PollBCD+ and AGV ${ }^{+i n s}$ famA DNAPs. Besides

180 PollBCD+ and AGV ${ }^{+i n s}$ famA DNAPs, 8-aa insertion was found solely in the famA

181 DNAP homolog of Vibrio phage ICP2 placed at the basal position of the clade of

182 PollBCD+ and $\mathrm{AGV}^{+i n s}$ famA DNAPs (Fig. 2). In the analyses of the second

183 alignment, $\mathrm{AGV}^{+ \text {ins }}$ famA DNAPs grouped together with an MLBP of $92 \%$ and a BPP

184 of 0.99 , excluding PollBCD+ sequences that formed a clade with an MLBP of $72 \%$

185 and a BPP of 0.66 (Fig. 2). The weak statistical support for the monophyly of

186 PollBCD+ sequences is not incongruent with their paraphyletic relationship

187 reconstructed in the global famA DNAP analysis (Fig. 1B). 


\section{AGV'+ins}
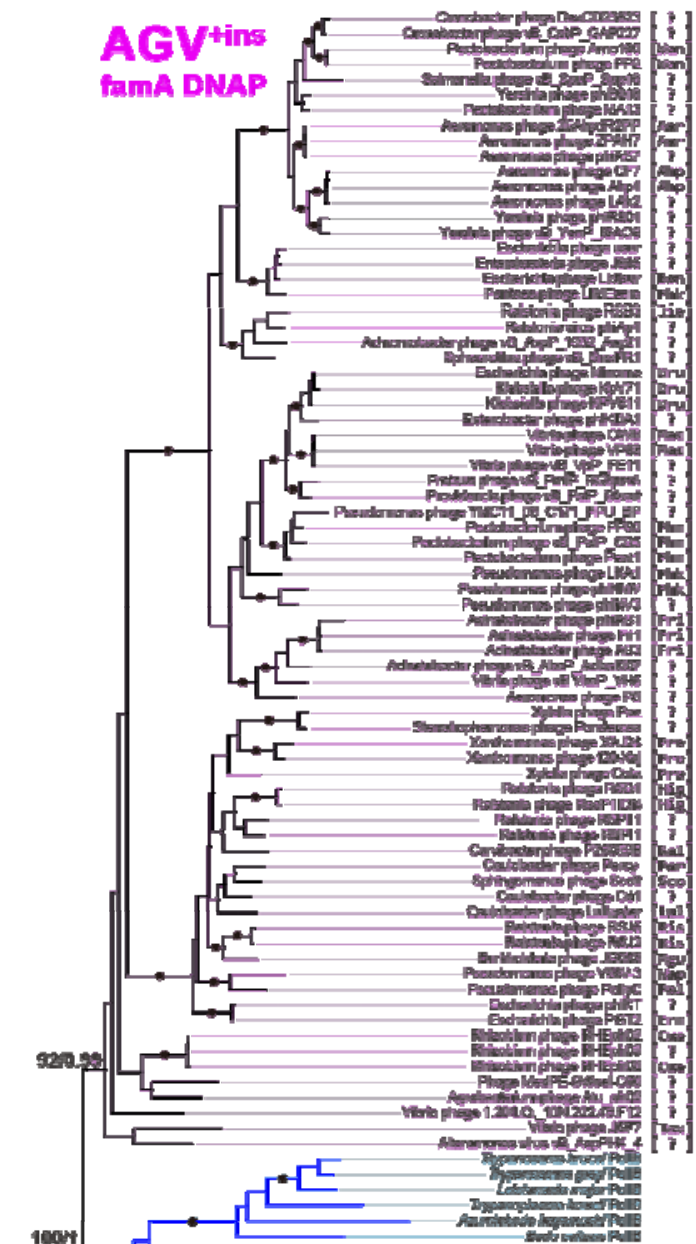

reas

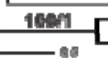

189

190

191

192

193

194

195

196

197

198

199

200

201

202

203

204

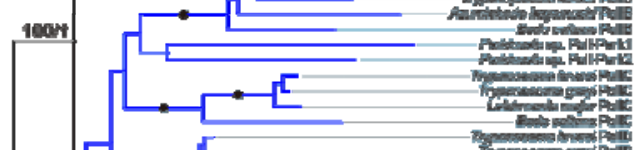

1

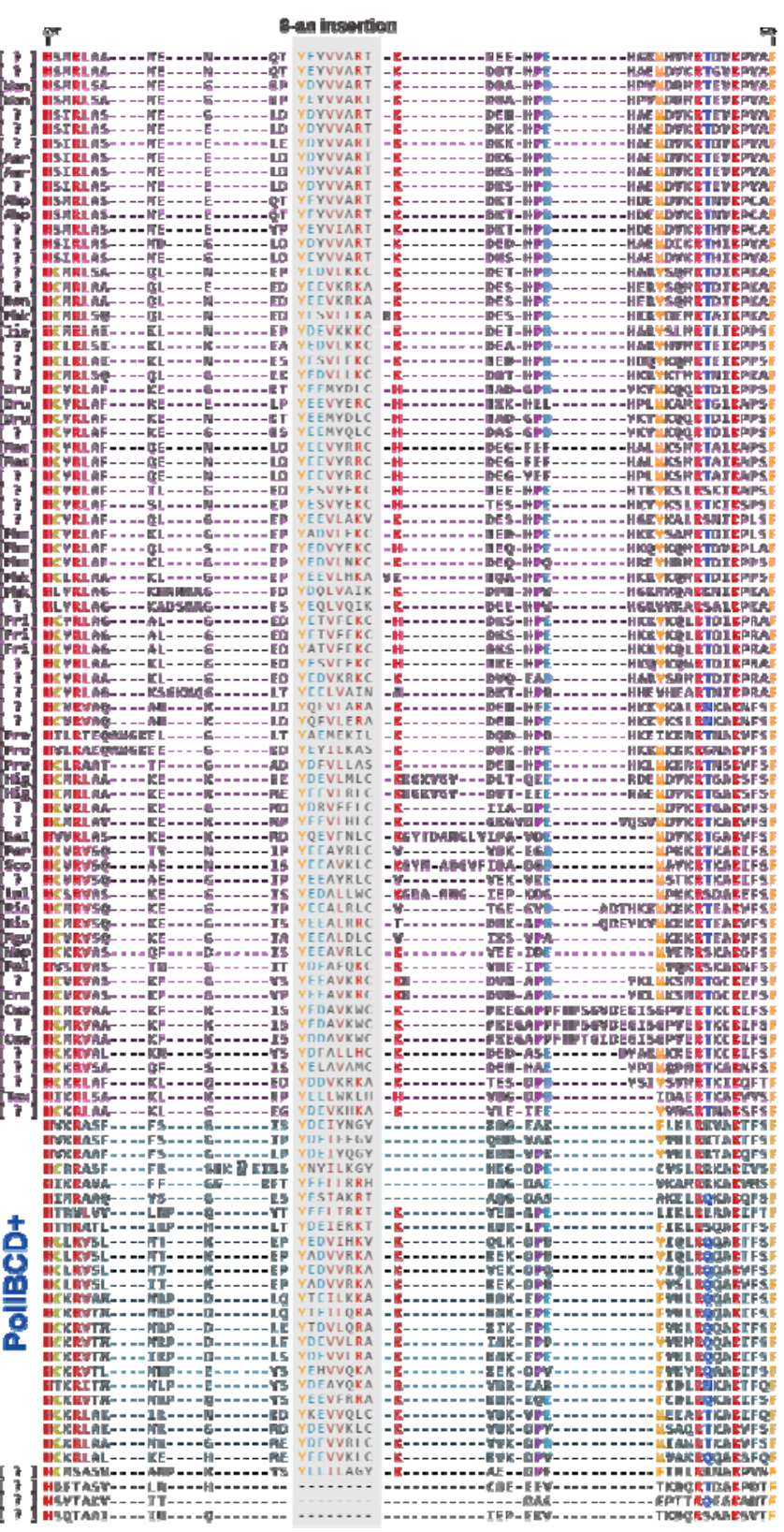

Fig. 2. Phylogenetic relationship among $74 \mathrm{AGV}^{\text {+ins }}$ famA DNAP and 24 PollBCD+ sequences that share a unique insertion of 8 amino acid residues (8-aa insertion). The tree topology and branch lengths inferred by the maximum likelihood (ML) method are shown on the left. ML bootstrap values (MLBPs) and Bayesian posterior probabilities (BPPs) for only the nodes critical to infer the origin of PollBCD+ are shown. As ML and Bayesian analyses reconstructed the essentially same tree topology, only BPPs for the selected nodes are presented. The nodes supported by an MLBP of $100 \%$ and a BPP of 1.0 are marked by dots. The genus names of the autographiviruses (and podoviruses), from which famA DNAPs were sampled, are given in brackets. Abbreviations are follows: Aer, Aerosvirus; Ahp, Ahphunavirus; Bon, Bonnellvirus; Cue, Cuernavacavirus; Dru, Drulisvirus; Erm, Ermolevavirus; Fri, Friunavirus; Hig, Higashivirus; Jia, Jiaoyazivirus; Kal, Kalppathivirus; Lul, Lullwatervirus; Mac, Maculvirus; Mgu, Mguuvirus; Nap, Napahaivirus; Per, Percyvirus; Phk, Phikmvvirus; Phm, Phimunavirus; Pol, Pollyceevirus; Pra, Pradovirus; Ris, Risjevirus; Sco, Scottvirus; Taw, Tawavirus; Wan, Wanjuvirus; ?, unclassified. The amino acid sequences of 8-aa insertions and their flanking regions are shown on the right. 8aa insertions are shaded in grey. The residues are colored according to their degrees of conservation. The amino acid residue numbers shown on the left and right edges of the 
205 alignment are based on the famA DNAPs of Cronobacter phage DevCD23823

206 (YP_009223394.1).

\section{Discussion}

208 The phylogenetic analyses of the global alignment of famA DNAPs (Figs. 1A 209 and $1 \mathrm{~B}$ ) and the second alignment rich in $\mathrm{AGV}^{\text {+ins }}$ famA DNAP homologs (Fig. 2) 210 consistently recovered the specific affinity between PollBCD+ and $A G V^{\text {+ins }}$ fam $A$ 211 DNAPs. These results strongly suggest that PollBCD+ in the extant kinetoplastids 212 and diplonemids can be traced back to a single autographivirus famA DNAP, 213 particularly the one with 8-aa insertion. In other words, PollBCD+ is a typical 214 example of non- $\alpha$-proteobacterial $\mathrm{mt}$ proteins established via lateral gene transfer. 215 Unfortunately, even the analyses of the second alignment, wherein the known 216 diversity of $A G V^{+i n s}$ famA DNAPs was covered, failed to pinpoint the exact origin of 217 PollBCD+ (Fig. 2). We might be able to find an $\mathrm{AGV}^{+ \text {ins }}$ famA DNAP homolog that 218 branches PollBCD+ sequences directly in a future phylogenetic study covering the 219 true diversity of phage famA DNAPs. In particular, we regard that autographivirus 220 famA DNAP genes in bacterial genomes are significant. To our knowledge, no 221 autographivirus has been reported to infect eukaryotes. Thus, the common ancestor 222 of kinetoplastids and diplonemids may have acquired the famA DNAP gene from a 223 lysogenic autographivirus in a bacterial genome. If so, the bacterial genomes 224 harboring $A G V^{+i n s}$ famA DNAP genes are critical to investigate the origin of 225 PollBCD+ at a finer level than that in the current study.

226 Members of classes Kinetoplastea and Diplonemea, together with Euglenida, 227 share another type of mitochondrion-localized famA DNAP, namely PollA (Harada et 228 al. 2020). It is reasonable to postulate that the common ancestor of the three 229 classes - most likely the ancestral euglenozoan—had established the ancestral 230 PollA. Although the origin of PollA has not been addressed explicitly, past studies 231 recovered the phylogenetic link between PollA and PolO, a type of famA DNAP 232 operated in the cytosol of eukaryotic cells. The original study reporting PollA, B, C, 233 and $\mathrm{D}$ in Trypanosoma brucei has hinted at the phylogenetic affinity between PollA 234 and PolO (Klingbeil et al. 2002). A recent phylogeny including famA DNAPs sampled 235 from eukaryotes and limited bacteria (Note that no phage homolog was included) 236 reconstructed a clade of PollA and Pol $\theta$ sequences with high statistical support 237 (Harada et al. 2020). The PollA-Pol $\theta$ affinity persisted even after the sampling of 
238 famA DNAPs from bacteria and phages was improved drastically in this study (Fig.

$2391 \mathrm{~A})$. We here propose that the ancestral PollA was likely derived from a Pol $\theta$

240 homolog followed by the change in subcellular localization from the cytosol to the

241 mitochondrion. Noteworthy, the evolutionary processes yielded PollA and PollBCD+,

242 both of which are mt proteins of non- $\alpha$-proteobacterial origin, are different

243 substantially from each other. The former emerged through the recycling of a pre-

244 existing eukaryotic protein while the latter is of phage origin (See above). The Pol $\theta$

245 origin of PollA is the best estimate from both past and current phylogenetic analyses

246 of famA DNAPs but alternative possibilities still need to be explored in future studies.

247 The repertories of mitochondrion-localized DNAPs in euglenozoans appeared

248 to be more complex than those in the majority of other eukaryotes in which a single

249 type of mitochondrion-localized DNAP (i.e. POP or Poly) seemingly operates. The

250 complexity in the repertory of DNAPs in euglenozoan mitochondria seems to

251 coincide with that in the structure of their mtDNAs (Lukeš et al. 2002; Roy et al.

252 2007; Spencer and Gray 2011; Dobáková et al. 2015; Yabuki et al. 2016; Burger and

253 Valach 2018). Nevertheless, it is unlikely that the non-a-proteobacterial background

254 is restricted to PollA and PollBCD+ among the proteins involved in mtDNA

255 maintenance. Rather, the machinery for mtDNA maintenance in the common

256 ancestor of kinetoplastids and diplonemids (and its descendants) are heavily

257 remodeled by both incorporating exogenous proteins via lateral gene transfer and

258 recycling the pre-existed nucleus-encoded proteins. The above conjecture can be

259 examined only after we identify the major proteins involved in DNA maintenance in

260 kinetoplastid/diplonemid mitochondria and their evolutionary origins.

\section{Materials \& Methods}

262 Global phylogeny of famA DNAPs

263 We searched for the amino acid (aa) sequences of bacterial and phage famA

264 DNAPs in the NCBI nr database as of March 6, 2020, by BLASTP using the

265 polymerase domain of Escherichia coli Poll (KHH06131.1; the portion corresponding 266 to the $491^{\text {st }}-928^{\text {th }}$ aa residues) as a query (Camacho et al. 2009; Sayers et al. 2020).

267 We retrieved the sequences matched to the query with $E$ values equal to or less than $2681 \times 10^{-4}$ and covered more than 200 aa in the polymerase domain. Note that the 
269 sequences derived from metagenome analyses were excluded from this study. The

270 redundancy within famA DNAP sequences was removed by cluster analysis using

271 CD-HIT v4.7 with a threshold of 40\% (Li and Godzik 2006; Fu et al. 2012). We finally

272 selected 119 and 327 aa sequences of phage and bacterial famA DNAPs,

273 respectively, for the downstream analyses (see below).

274 The bacterial and phage famA DNAP aa sequences (446 in total) were

275 aligned with those in eukaryotes (27 in total), namely (i) mitochondrion-localized

276 famA DNAPs in Kinetoplastea and Diplonemea (PollA, B, C, D, and Poll-dipl), (ii)

277 mitochondrion-localized famA DNAPs in animals and fungi (Poly), (iii) Pole localized

278 in the cytosol, (iv) mitochondrion and/or plastid-localized famA DNAPs in diverse

279 eukaryotes (POP), and (v) plastid-localized famA DNAPs in apicomplexan parasites

280 and their relatives (PREX). The aa sequences were aligned by MAFFT $v 7.455$ with

281 the L-INS-i model (Katoh and Standley 2013). Ambiguously aligned positions were

282 discarded manually, and gap-containing positions were trimmed by using trimAl v1.4

283 with the -gt 0.95 option (Capella-Gutiérrez et al. 2009). The final "global famA DNAP"

284 alignment comprised 473 sequences with 316 unambiguously aligned aa positions.

285 The final global famA alignment is provided as a part of the supplementary materials.

286 We subjected this alignment to the ML phylogenetic analysis by IQ-TREE v1.6.12

287 using the LG + $\Gamma+F+C 60+$ PMSF model (Nguyen et al. 2015; Wang et al. 2018).

288 The guide tree was obtained using the $L G+\Gamma+F$ model that was selected by

289 ModelFinder (Kalyaanamoorthy et al. 2017). The statistical support for each

290 bipartition in the ML tree was calculated by 100-replicate non-parametric bootstrap

291 analysis.

292 Phylogenetic analyses of an alignment rich in autographivirus famA

293 DNAPS

294 We retrieved 175 famA DNAP aa sequences of autographiviruses from the NCBI nr

295 database. The details of the survey were the same as described above. The 175

296 famA DNAPs were sampled from 99 members belonging to 57 genera and 76

297 unclassified members in the family Autographiviridae. The autographivirus famA

298 DNAPs were found to comprise two types based on the presence/absence of an

299 insertion of 8 aa residues (8-aa insertion; see above). The famA DNAPs with 8-aa

300 insertion ( $\mathrm{AGV}^{+ \text {ins }}$ famA DNAPs) appeared to be closely related to PollBCD+, 
301 mitochondrion-localized famA DNAPs in kinetoplastids (PollB, C, D, Poll-Perk1/2)

302 and that in diplonemids (Poll-dipl). The redundancy among the AGV ${ }^{\text {ins }}$ famA DNAPs

303 was reduced by cluster analysis using CD-HIT v4.7 with a threshold of $90 \%$. Finally,

304 we aligned the aa sequences of $74 \mathrm{AGV}^{+ \text {ins }}$ famA DNAPs, 24 PollBCD+, and famA

305 DNAPs of four members of Podoviridae by MAFFT v7.455 with the L-INS-i model.

306 Ambiguously aligned positions were discarded manually, and gap-containing

307 positions were trimmed by using trimAl v1.4 with the -gt 0.9 option. The final version

308 of the second alignment is provided as a part of the supplementary materials. The

309 final alignment containing 102 sequences with 581 unambiguously aligned aa

310 positions was subjected to both ML and Bayesian phylogenetic analyses. The ML

311 and ML bootstrap analyses were performed as described above. For Bayesian

312 analysis using Phylobayes v4.1, we run four Markov Chain Monte Carlo chains for

$313100,000$ cycles with burn-in of 25,000 (maxdiff $=0.09472)$ and calculated the

314 consensus tree with branch lengths and BPPs from the remaining trees (Lartillot et al.

315 2009). The amino acid substitution model was set to CAT + GTR in Phylobayes

316 analysis described above.

\section{Data availability}

318 The alignment datasets for phylogenetic analysis are available in supplementary

319 materials at

320 https://drive.google.com/drive/folders/1vpwh0MzYul_wjKmyutZIZR1MSmMZn5ca?us

$321 \quad \mathrm{p}=$ sharing.

322 Acknowledgments

323 This work was supported by the grants from the Japanese Society for Promotion of

324 Sciences awarded to Y. I. (numbers $18 \mathrm{KK} 0203$ and 19H03280).

Literature cited

326 Adriaenssens EM, Sullivan MB, Knezevic P, van Zyl LJ, Sarkar BL, et al. 2020.

327 Taxonomy of prokaryotic viruses: 2018-2019 update from the ICTV

328 bacterial and archaeal viruses subcommittee. Arch Virol. 165(5):1253-

329 1260. doi:10.1007/s00705-020-04577-8. 
330 Burger G, Valach M. 2018. Perfection of eccentricity: mitochondrial genomes of

331

332

333

334

335

336

337

338

339

340

341

342

343

344

345

346

347

348

349

350

351

352

353

354

355

356

357

358

359

360

361

362

363 diplonemids. IUBMB Life. 70(12):1197-1206. doi:10.1002/iub.1927.

Camacho C, Coulouris G, Avagyan V, Ma N, Papadopoulos J, et al. 2009. BLAST+: Architecture and applications. BMC Bioinformatics. 10:421:1-421:9. doi:10.1186/1471-2105-10-421.

Capella-Gutiérrez S, Silla-Martínez JM, Gabaldón T. 2009. trimAl: a tool for automated alignment trimming in large-scale phylogenetic analyses. Bioinformatics. 25(15):1972-1973. doi:10.1093/bioinformatics/btp348.

Dobáková E, Flegontov P, Skalický T, Lukeš J. 2015. Unexpectedly streamlined mitochondrial genome of the euglenozoan Euglena gracilis. Genome Biol Evol. 7(12):3358-3367. doi:10.1093/gbe/evv229.

El-Gebali S, Mistry J, Bateman A, Eddy SR, Luciani A, et al. 2019. The Pfam protein families database in 2019. Nucleic Acids Res. 47(D1):D427-D432. doi:10.1093/nar/gky995.

Fu L, Niu B, Zhu Z, Wu S, Li W. 2012. CD-HIT: accelerated for clustering the nextgeneration sequencing data. Bioinformatics. 28(23):3150-3152. doi:10.1093/bioinformatics/bts565.

Gabaldón T, Huynen MA. 2007. From endosymbiont to host-controlled organelle: the hijacking of mitochondrial protein synthesis and metabolism. PLoS Comput Biol. 3(11):2209-2218. doi:10.1371/journal.pcbi.0030219.

Gray MW. 2015. Mosaic nature of the mitochondrial proteome: implications for the origin and evolution of mitochondria. Proc Natl Acad Sci U S A. 112(33):10133-10138. doi:10.1073/pnas.1421379112.

Graziewicz MA, Longley MJ, Copeland WC. 2006. DNA polymerase $y$ in mitochondrial DNA replication and repair. Chem Rev. 106(2):383-405. doi:10.1021/cr040463d.

Harada R, Hirakawa Y, Yabuki A, Kashiyama Y, Maruyama M, et al. 2020. Inventory and evolution of mitochondrion-localized family A DNA polymerases in Euglenozoa. Pathogens. 9(4):257. doi:10.3390/pathogens9040257.

Hirakawa Y, Watanabe A. 2019. Organellar DNA polymerases in complex plastidbearing algae. Biomolecules. 9(4):140:1-140:12. doi:10.3390/biom9040140.

Janouškovec J, Tikhonenkov D V., Burki F, Howe AT, Kolísko M,et al. 2015. Factors mediating plastid dependency and the origins of parasitism in 
364

365

366

367

368

369

370

371

372

373

374

375

376

377

378

379

380

381

382

383

384

385

386

387

388

389

390

391

392

393

394

395

396

apicomplexans and their close relatives. Proc Natl Acad Sci U S A. 112(33):10200-10207. doi:10.1073/pnas.1423790112.

Jones P, Binns D, Chang HY, Fraser M, Li W, et al. 2014. InterProScan 5: genomescale protein function classification. Bioinformatics. 30(9):1236-1240. doi:10.1093/bioinformatics/btu031.

Jung GH, Leavitt MC, Hsieh JC, Ito J. 1987. Bacteriophage PRD1 DNA polymerase: evolution of DNA polymerases. Proc Natl Acad Sci U S A. 84(23):82878291. doi:10.1073/pnas.84.23.8287.

Kalyaanamoorthy S, Minh BQ, Wong TKF, Von Haeseler A, Jermiin LS. 2017. ModelFinder: fast model selection for accurate phylogenetic estimates. Nat Methods. 14(6):587-589. doi:10.1038/nmeth.4285.

Katoh K, Standley DM. 2013. MAFFT multiple sequence alignment software version 7: improvements in performance and usability. Mol Biol Evol. 30(4):772780. doi:10.1093/molbev/mst010.

Klingbeil MM, Motyka SA, Englund PT. 2002. Multiple mitochondrial DNA polymerases in Trypanosoma brucei. Mol Cell. 10(1):175-186. doi:10.1016/S1097-2765(02)00571-3.

Krasich R, Copeland WC. 2017. DNA polymerases in the mitochondria: a critical review of the evidence. Physiol Behav. 22(1):692-709. doi:10.1016/j.physbeh.2017.03.040.

Lartillot N, Lepage T, Blanquart S. 2009. PhyloBayes 3: a bayesian software package for phylogenetic reconstruction and molecular dating. Bioinformatics. 25(17):2286-2288. doi:10.1093/bioinformatics/btp368.

Lavigne R, Seto D, Mahadevan P, Ackermann HW, Kropinski AM. 2008. Unifying classical and molecular taxonomic classification: analysis of the Podoviridae using BLASTP-based tools. Res Microbiol. 159(5):406-414. doi:10.1016/j.resmic.2008.03.005.

Li W, Godzik A. 2006. Cd-hit: a fast program for clustering and comparing large sets of protein or nucleotide sequences. Bioinformatics. 22(13):1658-1659. doi:10.1093/bioinformatics/btl158.

Lukeš J, Guilbride DL, Votýpka J, Zíková A, Benne R, et al. 2002. Kinetoplast DNA network $\square$ : evolution of an improbable structure. Eukaryotic Cell. 1(4):495-502. doi:10.1128/EC.1.4.495. 
397 Moriyama T, Terasawa K, Sato N. 2011. Conservation of POPs, the plant organellar

398

399

400

401

402

403

404

405

406

407

408

409

410

411

412

413

414

415

416

417

418

419

420

421

422

423

424

425

426

427 DNA polymerases, in eukaryotes. Protist. 162(1):177-187. doi:10.1016/j.protis.2010.06.001. http://dx.doi.org/10.1016/j.protis.2010.06.001.

Nguyen LT, Schmidt HA, Von Haeseler A, Minh BQ. 2015. IQ-TREE: a fast and effective stochastic algorithm for estimating maximum-likelihood phylogenies. Mol Biol Evol. 32(1):268-274. doi:10.1093/molbev/msu300.

Roger AJ, Muñoz-Gómez SA, Kamikawa R. 2017. The origin and diversification of mitochondria. Curr Biol. 27(21):R1177-R1192. doi:10.1016/j.cub.2017.09.015.

Roy J, Faktorová D, Lukeš J, Burger G. 2007. Unusual mitochondrial genome structures throughout the Euglenozoa. Protist. 158(3):385-396. doi:10.1016/j.protis.2007.03.002.

Sayers EW, Beck J, Brister JR, Bolton EE, Canese K, et al. 2020. Database resources of the national center for biotechnology information. Nucleic Acids Res. 48(D1):D9-D16. doi:10.1093/nar/gkz899.

Spencer DF, Gray MW. 2011. Ribosomal RNA genes in Euglena gracilis mitochondrial DNA: fragmented genes in a seemingly fragmented genome. Mol Genet Genomics. 285(1):19-31. doi:10.1007/s00438-0100585-9.

Wang HC, Minh BQ, Susko E, Roger AJ. 2018. Modeling site heterogeneity with posterior mean site frequency profiles accelerates accurate phylogenomic estimation. Syst Biol. 67(2):216-235. doi:10.1093/sysbio/syx068.

Wang Z, Wu M. 2014. Phylogenomic reconstruction indicates mitochondrial ancestor was an energy parasite. PLoS One. 9(10):e110685. doi:10.1371/journal.pone.0110685.

Yabuki A, Tanifuji G, Kusaka C, Takishita K, Fujikura K. 2016. Hyper-Eccentric structural genes in the mitochondrial genome of the algal parasite Hemistasia phaeocysticola. Genome Biol Evol. 8(9):2870-2878. doi:10.1093/gbe/evw207. 